\title{
A retrospective study on the possible systematic inflammatory response markers to predict the prognosis of patients with bladder cancer undergoing radial cystectomy
}

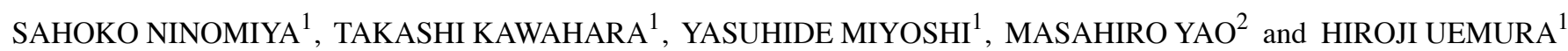 \\ ${ }^{1}$ Department of Urology and Renal Transplantation; ${ }^{2}$ Department of Urology, Reproduction Center, \\ Yokohama City University Medical Center, Yokohama, Kanagawa 2320024, Japan
}

Received February 18, 2020; Accepted July 1, 2020

DOI: $10.3892 / \operatorname{mco} .2020 .2117$

\begin{abstract}
Recent studies have shown that several inflammatory markers, including the neutrophil-to-lymphocyte ratio (NLR), monocyte-to-lymphocyte ratio (MLR), lymphocyte-to-platelet ratio (LPR), De Retis and prognostic nutritional index (PNI), can function as useful prognostic factors in a number of malignancies. The present study aimed to assess the most reliable inflammatory tumor marker in patients with bladder cancer who have undergone radical cystectomy. A total of 161 cases underwent radical cystectomy between 1994 and 2014 at Yokohama City University Medical Center (Yokohama, Japan). Of these patients, the 107 who had data for the NLR, MLR, LPR, De Retis and PNI were enrolled in the present study. The correlation between recurrence-free survival (RFS) or overall survival (OS) and inflammatory tumor markers were examined. Regarding the RFS, the only noteworthy finding was that a lower PNI was associated with a significantly poorer RFS compared with higher PNI $(\mathrm{P}=0.028)$. Regarding the OS, lower LPR and PNI were associated with a significantly poorer OS compared with higher values $(\mathrm{P}=0.048$ and $\mathrm{P}=0.036$, respectively). The present study revealed that a low PNI more accurately predicts a worse RFS and OS compared with other systemic inflammatory risk factors.
\end{abstract}

\section{Introduction}

Currently, there are no reliable biomarkers for predicting the prognosis of patients who undergo radical cystectomy for bladder cancer. Previous studies have shown that several inflammatory markers, including the neutrophil-to-lymphocyte ratio (NLR), monocyte-to-lymphocyte ratio (MLR), lymphocyte-to-platelet

Correspondence to: Dr Takashi Kawahara, Department of Urology and Renal Transplantation, Yokohama City University Medical Center, 4-57, Urafune-cho, Minami-ku, Yokohama, Kanagawa 2320024, Japan

E-mail: kawahara@yokohama-cu.ac.jp

Key words: neutrophil-to-lymphocyte ratio, lymphocyte-to-platelet ratio, monocyte-to-lymphocyte ratio, De Ritis, prognostic nutritional index ratio (LPR), De Retis and prognostic nutritional index (PNI), are raised as new prognostic factors for number of cancers (1-3).

The NLR, MLR, and LPR represent the ratios of lymphocytes to neutrophils, monocytes, and platelets (4). High values of these markers indicate a poor prognosis in the NLR. To the best of our knowledge, no other markers have been reported in bladder cancer (5). The detailed mechanisms underlying how these inflammatory cytokines are correlated with cancer progression remain unclear. A candidate mechanism involves malignant disease enhancing inflammation, thereby affecting regulatory $\mathrm{T}$ cells $(4,6)$. Cancer progression decreases the number of lymphocytes, which increases the NLR and MLR and decreases the LPR $(4,6)$.

Studies have shown that the PNI was correlated with the prognosis in several malignancies including lung and gastrointestinal cancers $(2,7)$. Systemic inflammation increased tumorigenesis and cancer progression, resulting in low albumin levels and immunosuppression (3). This phenomenon has been reported in colon, gastric, lung, and pancreatic cancers (8).

De Ritis is calculated as the aspartate transaminase (AST) levels divided by alanine transaminase (ALT) levels, as first reported by Fernando De Ritis in 1957 (8). This marker does not evaluate liver function but rather indicates the severity of liver dysfunction (8). In genitourinary cancer, a high De Ritis ratio was reported to be a poor prognostic marker in prostate, renal, and urothelial carcinoma. In other solid malignancies including breast and lung cancer, a high De Ritis ratio was also reported to be a poor prognostic marker (8).

The present study aimed to assess the most reliable inflammatory tumor marker in bladder cancer patients who have undergone radical cystectomy.

\section{Patients and methods}

A total of 161 cases underwent radical cystectomy between April 1994 and April 2014 at Yokohama City University Medical Center (Yokohama, Japan). The median and mean follow-up periods were 29.2 and 39.4 \pm 31.4 months and all patients were $>18$ years. Of these patients, the 107 who had data for the NLR, MLR, LPR, De Ritis and PNI were enrolled in the present study (Table I). The PNI is calculated using the albumin and lymphocyte count with the following formula: $\mathrm{PNI}=(10 \mathrm{x}$ albumin $)+$ 
(0.005 x total lymphocyte count) (7). A total of 31 (29.2\%) cases received cisplatin-based neo-adjuvant systemic chemotherapy. Laboratory data, including complete blood cell count (CBCs) and albumin, AST and ALT levels, were obtained a few days before surgery. CBCs included the number of blood cells, platelets and white blood cells with differentiation. None of the patients showed systemic inflammation or blood disease at the time of the blood test. The criteria of systemic inflammation were determined as fever and elevation of serum C-reactive protein (CRP) levels. In the present cohort, none of the patients had fever and CRP elevation. The median value was used as the cut-off value of each factor as follows: NLR, 1.00; MLR, 0.24: LPR, 73.9; PNI, 44.0 and De Ritis, 1.1. Receiver operator characteristic (ROC) curves were plotted to show candidate cutoff points. The candidate cutoff points and the area under the ROC (AUROC) are shown in Table II. Postoperative complications were assessed using the Clavien-Dindo Grading System and the existence of surgical complications was defined by the presence of complications classified as Clavien-Dindo Grade I or higher (9). The Institutional Review Board of Yokohama City University Medical Center (Yokohama, Japan) approved the present study (approval no. D1507018) and written informed consent was waivered due to the nature of the retrospective observational study without patient identification.

The preoperative NLR, MLR LPR, De Ritis and PNI were examined as prognostic factors for recurrence-free survival (RFS) and overall survival (OS). A Kaplan-Meier product limit estimate was used to estimate the OS and RFS. The log-rank test was performed to compare each risk factor. Values between neoadjyuvant chemotherapy (NAC) and non-NAC groups were compared. $\mathrm{P}<0.05$ was considered to indicate a statistically significant difference. Statistical analyses were performed using the GraphPad Prism 7.0 (GraphPad Software, Inc.).

\section{Results}

Regarding the RFS, the only noteworthy finding was that a lower PNI was associated with a significantly lower RFS compared with higher PNI ( $\mathrm{P}=0.028)$. Regarding the OS, lower LPR and PNI were associated with significantly lower OS compared with higher values (more than median value) ( $\mathrm{P}=0.048$ and $\mathrm{P}=0.036$, respectively). When the cutoff point was set as the median value, the other inflammatory markers showed no significant association with either the RFS or OS (Fig. 1). When the cutoff point was set using the ROC curve, a lower PNI was associated with significantly lower RFS and OS ( $\mathrm{P}=0.0143$ and $\mathrm{P}=0.0216$, respectively; Fig. 2). Based on these findings, De Ritis was evaluated as a prognostic risk factor after radical cystectomy. No differences were observed between these systemic inflammatory markers and the pT stage, lymph node metastasis, age, neo-adjuvant systemic chemotherapy or sex (Table SI).

\section{Discussion}

The current strategy for the treatment muscle-invasive bladder cancer is based on staging using imaging modalities (3). For non-metastatic muscle-invasive bladder cancer, radical cystectomy is the gold standard treatment (3). While radical cystectomy has proven effective, the recurrence rate is still not
Table I. Patient characteristics.

Characteristics

No. of subjects (\%)

Age, years

$<65$

$42(39.3)$

$\geq 65$

Prognosis

Alive

$79(73.8)$

Death

Neo-adjuvant chemotherapy

Yes

No

Recurrence

Pathological T stage

$\leq 2$

$\geq 3$

Pathological N stage

Positive

Postoperative complications

$21(19.6)$

$\mathrm{n}=107$.

Table II. Candidate cutoff points determined using ROC analyses.

A, Overall survival

\begin{tabular}{lcc}
\hline Marker & AUROC & Cutoff value \\
\hline NLR & 0.6044 & 2.38 \\
MLR & 0.5136 & 0.18 \\
LPR & 0.5927 & 71.60 \\
PNI & 0.6731 & 47.90 \\
De Ritis & 0.6022 & 1.30 \\
\hline
\end{tabular}

$\mathrm{B}$, Recurrence free survival

\begin{tabular}{lcc}
\hline Marker & AUROC & Cutoff value \\
\hline NLR & 0.5123 & 2.38 \\
MLR & 0.5381 & 0.25 \\
LPR & 0.5135 & 71.60 \\
PNI & 0.6605 & 47.90 \\
De Ritis & 0.5561 & 1.08 \\
\hline
\end{tabular}

ROC, receiver operating characteristic; AUROC, area under the ROC; NLR, neutrophil-to-lymphocyte ratio; MLR, monocyte-to-lymphocyte ratio; LPR, lymphocyte-to-platelet ratio; PNI, prognostic nutritional index.

entirely sufficient (4). Thus, preoperative risk management is necessary. Previous studies have revealed the usefulness of inflammatory markers in several solid malignancies, including bladder cancer (2). 

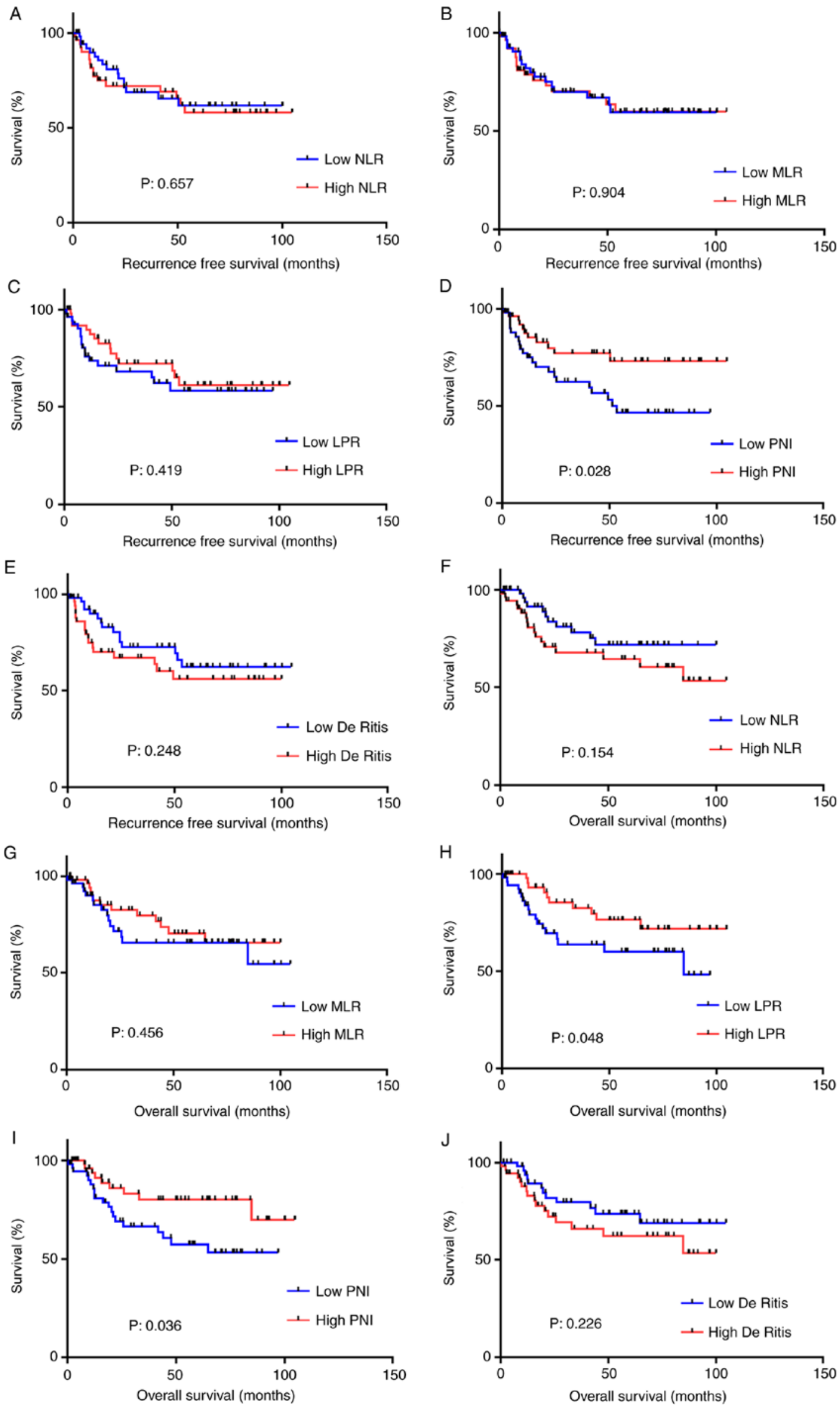

Figure 1. Overall survival in each prognostic factor. Recurrence-free survival of (A) NLR, (B) MLR, (C) LPR, (D) De Ritis and (E) PNI and overall survival of (F) NLR, (G) MLR and (H) LPR, (I) De Ritis and (J) PNI using the cutoff points as median value. NLR, neutrophil-to-lymphocyte ratio; MLR, monocyte-to-lymphocyte ratio; LPR, lymphocyte-to-platelet ratio; PNI, prognostic nutritional index. 

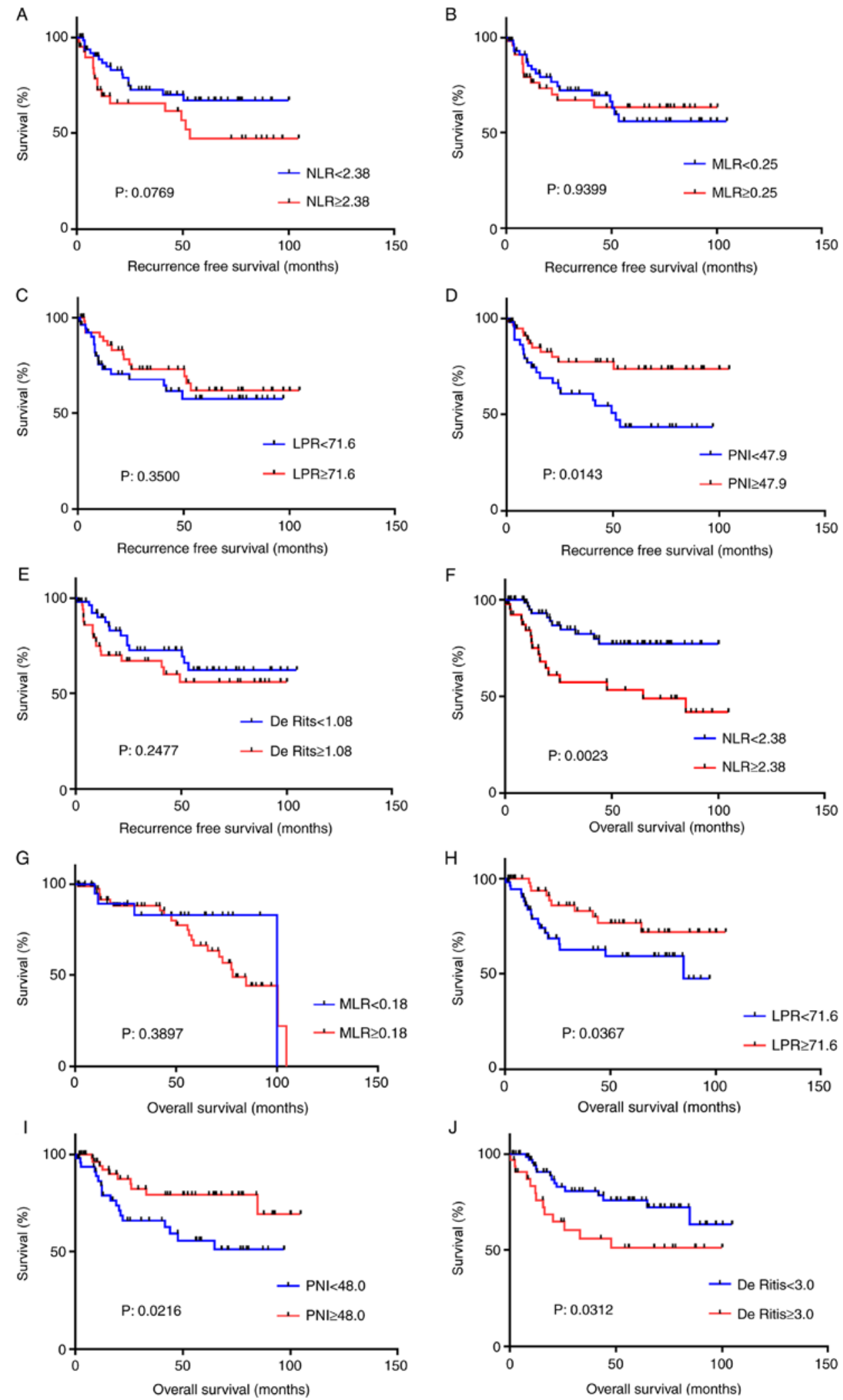

Figure 2. Recurrence-free survival in each prognostic factor. The recurrence-free survival according to (A) NLR, (B) MLR, (C) LPR, (D) PNI, and (E) De Ritis . Overall survival according to (F) NLR, (G) MLR, (H) LPR, (I) PNI and (J) De Ritis, as determined using candidate cutoff points identified in a receiver operator characteristic curve analysis. NLR, neutrophil-to-lymphocyte ratio; MLR, monocyte-to-lymphocyte ratio; LPR, lymphocyte-to-platelet ratio; PNI, prognostic nutritional index.

The present study examined the effectiveness of systemic inflammatory markers, including NLR, PNI, MLR, and LPR for predicting prognosis of bladder cancer. In addition, the utility of De Ritis was evaluated as a prognostic risk factor after radical cystectomy. A lower PNI was found to be associated with a significantly poorer prognosis in both the RFS and OS. This suggested that albumin may play a role in bladder cancer progression, in addition to lymphocytes. Therefore, a lower 
preoperative PNI predicted an increased risk of recurrence, which suggested that several adjuvant or neo-adjuvant therapies should be performed in combination with radical cystectomy.

Onodera et al revealed that a PNI of $>45$ denoted a low risk of surgical complications, a PNI of 40-45 denoted a high risk of surgical complications and a PNI of $<40$ suggested that surgery should be avoided (10). A recent study showed that the PNI was correlated with the prognosis of several malignancies including lymphoma and pancreas cancer $(11,12)$. Systemic inflammation increases tumorigenesis and cancer progression, resulting in low albumin levels and immunosuppression (7). This phenomenon has been reported in colon, gastric, lung, and pancreatic cancers (7). Although nutrition plays an important role in patients undergoing radical cystectomy, no studies have reported a correlation between the PNI and survival and recurrence (13).

Several limitations associated with the present study warrant mention. First, the present study initially used median values and values determined based on ROC curves as candidate cutoff points. Until now, no established cut-off point has been determined. Although median value might have included some good cutoff points, the median value cutoff points determined from ROC curves were ultimately used for analysis. The PNI demonstrated significance using both sets of cutoff points. Second, the number of patients was relatively small. Therefore, further studies are needed in order to validate the superiority of the PNI to other markers. Third, patients from 1994 to 2014 were included. Although there have been improvements in muscle invasive bladder cancer treatment such as development of gemcitabine and cisplatin treatment, there have been no dramatic changes until 2018. Until 2018, immune-checkpoint inhibitors were not permitted for daily clinical use. Furthermore, none of the patients in the present cohort received pembrolizumab.

In conclusion, the present study revealed that a low PNI more accurately predicted lower RFS and OS compared with other systemic inflammatory risk factors.

\section{Acknowledgements}

Not applicable.

\section{Funding}

This study was supported by KAKENHI grants (grant no. 16K20152) from the Ministry of Education, Culture, Sports, Science and Technology of Japan and grant for 2016-017 Research Development Fund (grant no. WJ2810) of Yokohama City University.

\section{Availability of data and materials}

The datasets generated and/or analyzed during the current study are not publicly available due to ethical restrictions but are available from the corresponding author on reasonable request.

\section{Authors' contributions}

SN and TK drafed the manuscript and performed stastical analysis and interpretation of data. SN, TK, YM, MY and $\mathrm{HU}$ acquired the data. All authors read and approved the final manuscript.

\section{Ethics approval and consent to participate}

The present study was approved by the Institutional Review Board (IRB) of Yokohama City University Medical Center. This study is a retrospective observational study and the IRB did not require written informed consent blinded to patients' identification.

\section{Patient consent for publication}

Not applicable.

\section{Competing interests}

The authors declare that they have no competing interests.

\section{References}

1. Potretzke A, Hillman L, Wong K, Shi F, Brower R, Mai S, Cetnar JP, Abel EJ and Downs TM: NLR is predictive of upstaging at the time of radical cystectomy for patients with urothelial carcinoma of the bladder. Urol Oncol 32: 631-636, 2014.

2. Tokunaga R, Sakamoto Y, Nakagawa S, Miyamoto Y, Yoshida N, Oki E, Watanabe $\mathrm{M}$ and Baba H: Prognostic nutritional index predicts severe complications, recurrence, and poor prognosis in patients with colorectal cancer undergoing primary tumor resection. Dis Colon Rectum 58: 1048-1057, 2015.

3. Ohno Y: Role of systemic inflammatory response markers in urological malignancy. Int J Urol 26: 31-47, 2019.

4. Sarraf KM, Belcher E, Raevsky E, Nicholson AG, Goldstraw P and Lim E: Neutrophil/lymphocyte ratio and its association with survival after complete resection in non-small cell lung cancer. J Thorac Cardiovasc Surg 137: 425-428, 2009.

5. Kawahara T, Furuya K, Nakamura M, Sakamaki K, Osaka K, Ito H, Ito Y,Izumi K, Ohtake S, Miyoshi Y, et al: Neutrophil-to-lymphocyte ratio is a prognostic marker in bladder cancer patients after radical cystectomy. BMC Cancer 16: 185, 2016

6. Ohtake S, Kawahara T, Kasahara R, Ito H, Osaka H, Hattori Y, Teranishi JI, Makiyama K, Mizuno N, Umemoto S, et al: Pretreatment neutrophil-to-lymphocyte ratio can predict the prognosis in bladder cancer patients who receive gemcitabine and nedaplatin therapy. Biomed Res Int 2016: 9846823, 2016.

7. Feng JF, Huang Y and Chen QX: Preoperative platelet lymphocyte ratio (PLR) is superior to neutrophil lymphocyte ratio (NLR) as a predictive factor in patients with esophageal squamous cell carcinoma. World J Surg Oncol 12: 58, 2014.

8. Botros M and Sikaris KA: The de ritis ratio: The test of time. Clin Biochem Rev 34: 117-130, 2013.

9. Dindo D, Demartines N and Clavien PA: Classification of surgical complications: A new proposal with evaluation in a cohort of 6336 patients and results of a survey. Ann Surg 240: 205-213, 2004.

10. Onodera T, Goseki N and Kosaki G: Prognostic nutritional index in gastrointestinal surgery of malnourished cancer patients. Nihon Geka Gakkai Zasshi 85: 1001-1005, 1984 (In Japanese).

11. Go SI, Park S, Kang MH, Kim HG, Kim HR and Lee GW: Clinical impact of prognostic nutritional index in diffuse large $B$ cell lymphoma. Ann Hematol 98: 401-411, 2019.

12. Nakagawa S, Yamashita YI, Umezaki N, Yamao T, Okabe H, Imai K, Nitta H, Hashimoto D, Chikamoto A and Baba H: Serum marker score based on prognostic nutrition index, carcinoembryonic antigen, and carbohydrate antigen 19-9 is associated with recurrence for patients undergoing surgery for pancreatic ductal adenocarcinoma. Pancreas 47: 1130-1134, 2018.

13. Miyake M, Morizawa Y, Hori S, Marugami N, Iida K, Ohnishi K, Gotoh D, Tatsumi Y, Nakai Y, Inoue T, et al: Integrative assessment of pretreatment inflammation-, nutrition-, and muscle-based prognostic markers in patients with muscle-invasive bladder cancer undergoing radical cystectomy. Oncology 93: 259-269, 2017.

This work is licensed under a Creative Commons Attribution-NonCommercial-NoDerivatives 4.0 International (CC BY-NC-ND 4.0) License. 